\begin{tabular}{|c|c|c|c|}
\hline Eiszeitalter $u$. Gegenwart & $\mathbf{2 9}$ & $\begin{array}{c}115-121 \\
3 \mathrm{Abb} .\end{array}$ & Hannover 1979 \\
\hline
\end{tabular}

\title{
Glaziale Uabertiefung und postglaziale Talverschüttung im Etschtal im Raum von Trient (Italien)
}

\author{
Giulio Antonio Venzo *)
}

Glacial erosion (overdeepening), valley, isobathe (quaternary basis), eustasy, upper pleistocene, borehole section, Trentino Alto Adige (Adige Valley).

K urzf assung: Aus der Schichtfolge von vier Brunnenbohrungen, die im Etschtal im Raum von Trient zur Ausführung gelangten, geht hervor, daß hier nach Rückzug der Würmgletscher im Stau der Schuttkegel von Seitentälern ein postglaziales Seebecken bestand.

Die Ergebnisse von geoelektrischen, an den Bohrungen geeichten Tiefensondierungen gestatten, zumindest in großen Zügen, die Rekonstruktion der Morphologie des Grundgebirges im Liegenden der postglazialen Talfüllung sowie die Ermittlung des Höchstbetrages der Taleintiefung; die Felssohle liegt in $267 \mathrm{~m}$ unter der jetzigen Talsohle $(193 \mathrm{~m}$ üb. NN) d. h. $74 \mathrm{~m}$ unter dem gegenwärtigen Meeresspiegel.

Aus den stratigraphischen, sedimentologischen und geophysikalischen Befunden geht hervor, daß im Quartär, zumindest im Würm und im Postglazial, kausale Beziehungen zwischen den Meeresspiegelschwankungen und der geomorphologischen Entwicklung des Etschtales, auch im inneralpinen Bereich nördlich von Trient, bestanden.

\section{[Sovraescavazione glaciale e riempimento postglaciale nella valle dell'Adige presso Trento (Italia)]}

Ri as s u n to: Le stratigrafie di quattro pozzi per acqua perforati meccanicamente nella valle dell'Adige nei dintorni di Trento, indicano l'esistenza, successiva al ritiro dei ghiacciai würmiani, di un bacino lacustre post glaciale determinato dagli sbarramenti di conoidi torrentizi di valli laterali.

Prospezioni geoelettriche, tarate sui sondaggi meccanici, hanno consentito di ricostruire, almeno nelle grandi linee, la morfologia del bed rock sotto i depositi postglaciali di fondovalle; e di accertare la profondità massima dell'incisione valliva a $267 \mathrm{~m}$ sotto la superfice topografica del talweg attuale (q. m 193), vale a dire $74 \mathrm{~m}$ al di sotto del livello attuale del mare.

I dati stratigrafici, sedimentologici e geofisici emersi dalle indagini suddette indicano che nel Quaternario, almeno nel Würm e nel post Würm vi furono relazioni di causa ed effetto tra oscillazioni del livello marino ed evoluzione geomorfologica della valle dell'Adige anche nel tratto alpino a nord di Trento.

\section{[Glacial Overdeepening and Postglacial Upfilling in the Adige Valley in the Surroundings of Trento (Italy)]}

A b t r a ct: The stratigraphic series of four water wells drilled in the Adige River valley on the outskirts of Trento, show the existence, immediately subsequent to the drawing back of the würmian glaciation, of a post-glacial lacustrine basin, determined by the barrings caused by lateral alluvial cones.

Geoelectrical surveys, previously set on the borings, have made it possible to outline the morphology of the bed-rock under the post-würmian Pleistocene deposits; and to ascertain the maximum depth of the glacial channel found at $267 \mathrm{~m}$ under the topographic surface (h. 193), which means $74 \mathrm{~m}$ below the present sea level.

*) Anschrift des Verfassers: Prof. Dott. G. A. V e n z o, Ordinario di Geologia nell’Università degli Studi di Trieste, Piazza Europa, I 34127 Trieste. 

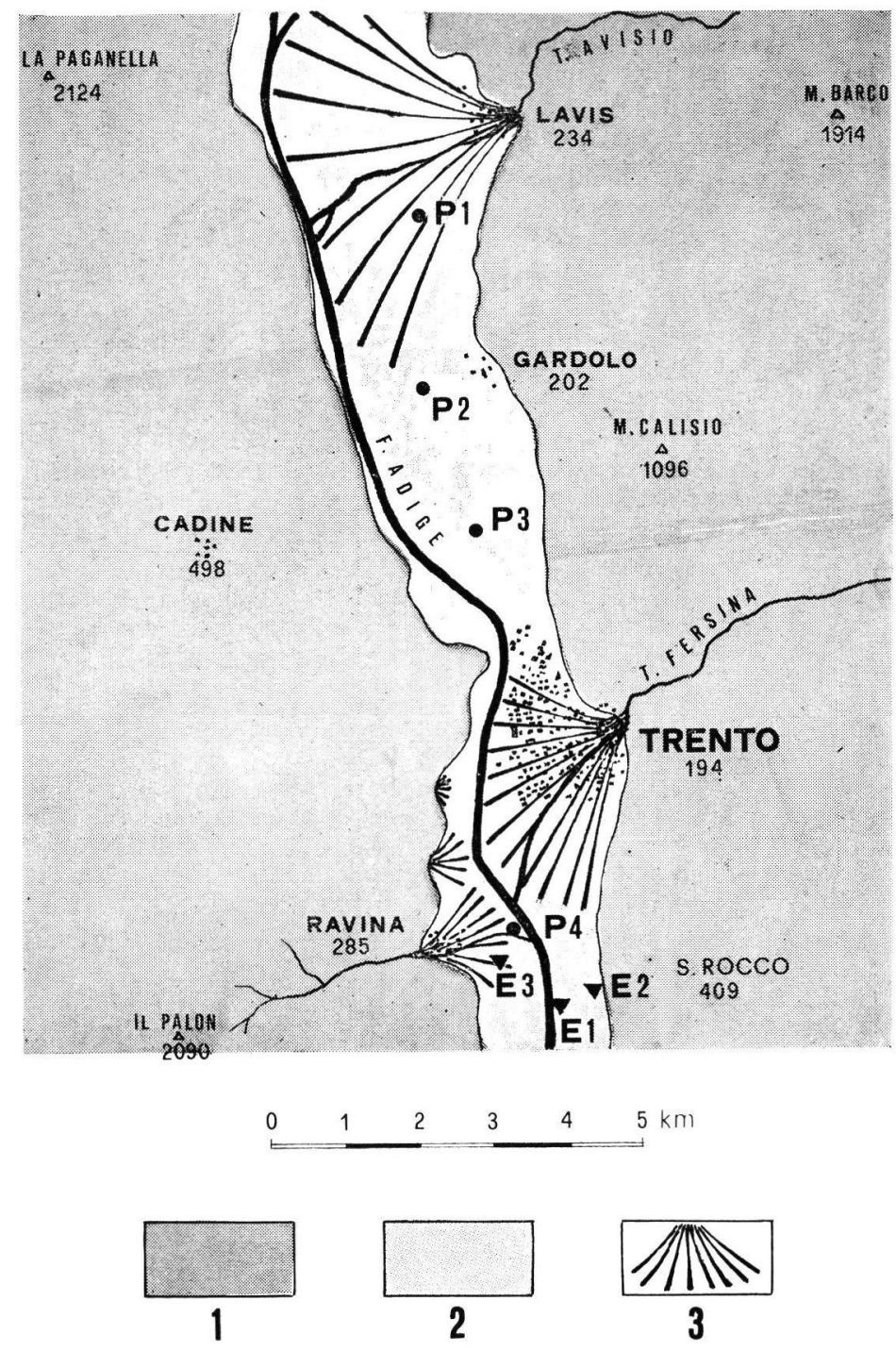

Abb. 1: Das Etschtal im Raum von Trient (1 = Präquartäre Ablagerungen; 2 = Postglaziale Talverschüttung; 3 = Schwemmkegel; $\mathrm{P}=$ Bohrbrunnen; $\mathrm{E}=$ Geoelektrische Meßpunkte). 
The sedimentologic, stratigraphic and geophysical data arising from the aforesaid investigations, show that in the Pleistocene, at least in the Würm and in post-Würm, there have been relations of cause and effect between oscillations in sea level and geomorphological evolution of the Adige River valley, also in the north of Trento.

\section{Einleitung}

Mit vorliegendem Beitrag erhebt der Autor keineswegs den Anspruch, die zahlreichen Fragen zu klären, die mit der geomorphologischen Entwicklung des Etschtales im Quartär in Zusammenhang stehen und noch einer befriedigenden Antwort harren. Er beschränkt sich darauf, die Ergebnisse neuer Untersuchungen mitzuteilen und daran einige Deutungsversuche zu knüpfen, in der Absicht, einen, wenn auch nur bescheidenen Beitrag zur Kenntnis des bedeutendsten übertieften Tales der Alpensüdseite zu liefern.

\section{Die postglaziale Talverschüttung}

Im Jahre 1957 wurden in der Industriezone am nördlichen Stadtrand von Trient einige Brunnenbohrungen niedergebracht. Die tiefste (P3 der Abb. 1 und 2) mit Ansatzpunkt in $190 \mathrm{~m}$ üb. NN erreichte eine Teufe von $193 \mathrm{~m}$, ohne auf die Felssohle des Tales zu stoßen. Ich hatte Gelegenheit, die Abteufung dieser bisher mit Abstand tiefsten Bohrung des gesamten Etschtales zu verfolgen, verfüge über Kerne und Schichtverzeichnis und habe die erschlossene Schichtfolge bereits vor Jahren einer vorwiegend sedimentpetrographischen Untersuchung unterzogen (G. A. Venzo 1957).

Zeitlich sowohl vor wie nach der erwähnten Tiefbohrung wurden im Etschtal zahlreiche weitere Brunnenbohrungen niedergebracht. Es handelt sich vorwiegend um Flachbohrungen oder um Bohrungen von beschränkter Teufe, über die meist keine oder nur unzuverlässige Unterlagen verfügbar sind. Nur einige dieser Bohrungen, wie P1, P2, P3 und P4 (vgl. Abb. 2) erwiesen sich immerhin auf Grund der verhältnismäßig bedeutenden Länge und der durchteuften Schichtserie als ausreichend aufschlußreich, so daß ihre Ergebnisse zur Rekonstruktion der Schlußphasen der Talverschüttung mit herangezogen werden konnten.

Die vier vorliegend berücksichtigten Bohrungen haben nachstehende Schichtfolgen erschlossen:

Bohrung P1 (Höhenlagedes Ansatzpunktes $200 \mathrm{~m}$ üb. N N)

0,00-55,00 m (größte erreichte Teufe): sehr grobkörnige steinig-kiesige, schwach sandige, vorwiegend aus Porphyrkomponenten bestehende Alluvionen mit vereinzelten schmächtigen Sandlagen.

Bohrung P 2 (Höhenlagedes Ansatzpunktes $195 \mathrm{~m}$ üb. N N)

0,00-7,30 m: schluffiger Sand mit Torf; 7,30-13,30 m: sandiger Kies mit spärlichen Einlagerungen von Fein- und Mittelsand; 13,30-23,80 m: schluffiger Ton mit Torf; 23,80-37,00 m (größte Teufe): sandiger Kies.

Bohrung P 3 (Höhenlagedes Ansatzpunktes $190 \mathrm{~m}$ üb. NN)

0,00-7,30 m: sandiger, quarz- und glimmerhaltiger Schluff; 7,30-13,40 m: polymikter sandiger Kies (Porphyr-, Kristallin-, Kalk- und Dolomitgerölle); 13,40-15,40 m: toniger, stark glimmerhaltiger Schluff; 15,40-50,00 m: Sand mit Torflagen; 50,00 bis 99,00 m: toniger Schluff; 99,00-103,00 m: gleichförmiger Feinschotter aus schwach gerundeten, vorwiegenden Kristallingeröllen; 103,00-193,00 m (größte Teufe): toniger Schluff, entsprechend jenem in 50,00-99,00 m Tiefe. 


\section{Bohrung P 4 (Höhenlage des Ansatzpunktes 190 m üb. NN)}

0,00-31,00 m: grobkörnige steinig-kiesige Alluvionen; $31,00-35,00 \mathrm{~m}$ : sandiger Kies mit spärlichen sandig-schluffigen Lagen; 35,00-50,00 m (größte Teufe): sandiger Kies.

Die Bohrungen P1 und P4 liegen auf Schwemmkegeln, die Bohrung P2 am Rande des durch die Bohrung P1 erschlossenen Kegels, die Bohrung P3 zwischen dem großen, vom Wildbach Avisio aufgeschütteten Kegel von Lavis im Norden und den Kegeln des Wildbaches Fersina bzw. von Ravina im Süden, auf denen sich die Stadt Trient erhebt (vgl. Abb. 1).

NOR D

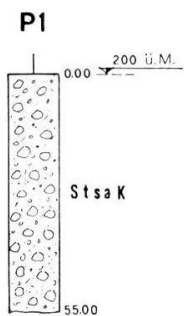

P2

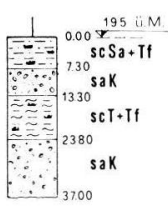

P3

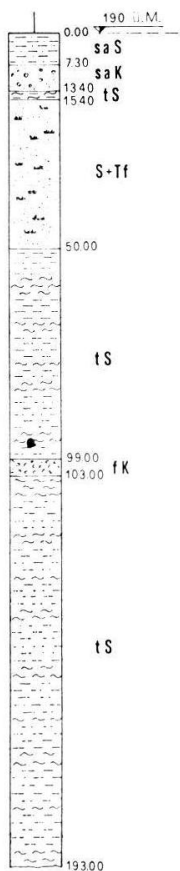

SU 0

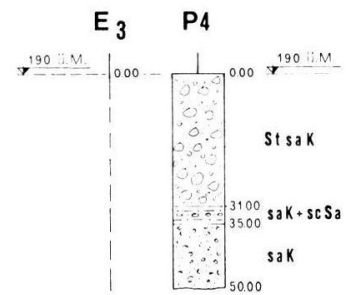

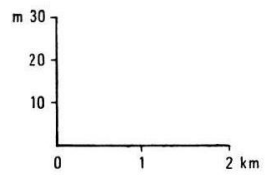

Abb. 2: Schichtfolge und Lage der vier Brunnenbohrungen $P_{1}, P_{2}, P_{3}, P_{4}$ und des $E_{3}$ geoelektrischen Meßpunktes im Raum von Trient (StsaK = Steine und sandiger Kies; saK = sandiger Kies; $\mathrm{fK}=$ Feinkies; $\mathrm{Sa}=$ Sand; $\mathrm{scSa}=$ schluffiger Sand; $\mathrm{saS}=$ sandiger Schluff; $\mathrm{tS}=$ toniger Schluff; scT $=$ schluffiger Ton; $\mathrm{Tf}=$ Torf; FS = Felssohle). 
Die Bohrungen P1 und P4 haben Schichtfolgen erschlossen, die sich in sedimentpetrographischer Hinsicht und auf Grund der Körnung als Wildbachablagerungen erweisen (Steine, Grobkies, untergeordnet Sand). Es folgt daraus, daß die Aufschüttung des Tales abschnittsweise Schwemmkegeln von Wildbächen zuzuschreiben ist. Dies trifft mit Sicherheit für die der erbohrten Schichtmächtigkeit entsprechenden Schlußphase der Talentwicklung zu; doch ist anzunehmen, daß Kegelbildungen bereits zu Beginn der letzten Nacheiszeit einsetzten.

Die Bohrung P2 hat, in Übereinstimmung mit ihrer Lage am Südrand des Kegels von Lavis, eine mehrfach verzahnte Folge von Schwemmkegel- (sandiger Kies) und Seeablagerungen (schluffiger Ton mit Torf) erschlossen.

Wesentlich aufschlußreicher, auch wegen der bedeutenderen Teufe, ist die Bohrung P3.

Die Schichtfolge besteht in tieferen Anteilen aus typischen See-Ablagerungen (toniger Schluff), in höheren aus Flußablagerungen (sandiger Kies). Den allmählichen Übergang bildet Feinsand mit Torflagen. Der $143 \mathrm{~m}$ mächtigen einförmigen tonigen Schluff-Folge ist nur in 99 bis $103 \mathrm{~m}$ Tiefe kantiger Feinkies eingelagert ${ }^{2}$ ).

Da weder Moränen noch andere Ablagerungen durchteuft wurden, die auf eine Unterbrechung des fluviatil-limnischen Sedimentationszyklus hinweisen würden, darf angenommen werden, daß die gesamte erschlossene Schichtfolge dem Postglazial angehört.

Auch die Bohrung P3 hat die felsige Talsohle nicht erreicht; es ist daher nicht bekannt, ob die Seesedimente unmittelbar dem Grundgebirge auflagern oder ob, was näherliegend erscheint, auf der Felssohle, d. h. zwischen ihr und der erbohrten fluviatil-limnischen Folge, Moränenabsätze erhalten sind.

\section{Die Übertiefung des Tales}

Mit finanzieller Unterstützung des Consiglio Nazionale delle Ricerche veranlaßte ich im Jahre 1976 die Durchführung geophysikalischer Untersuchungen, und zwar geoelektrischer Messungen, im Raum unmittelbar nördlich und südlich von Trient. Zweck der Sondierungen war die Ermittlung des Verlaufes der durch Lockergesteine verschütteten Felssohle sowie des Betrages der Úbertiefung des Tales. Diese muß nach den Ergebnissen einer Bohrung, der P3, die das Grundgebirge nicht erreicht hat, sicherlich unter den gegenwärtigen Meeresspiegel hinabreichen (vgl. Abb. 2).

Nur im Süden der Stadt gelang im Bereich der Meßstellen E1, E2 und E3 (vgl. Abb. 1) der Nachweis des Felsuntergrundes. Er unterscheidet sich durch wesentlich höheren Widerstand von der quartären Überlagerung, deren Mächtigkeit sich daher mit befriedigender Genauigkeit wie folgt feststellen ließ: E1 $=198 \mathrm{~m}, \mathrm{E} 2=105 \mathrm{~m}, \mathrm{E} 3=267 \mathrm{~m}$.

Die geophysikalischen Untersuchungen haben weiterhin aber auch die Rekonstruktion der Felssohle ermöglicht (vgl. Abb. 3). Wenngleich die Lage derselben nur annäherungsweise ermittelt werden konnte, kommt der Rekonstruktion doch beachtliches Interesse zu, $\mathrm{da}$ ihr zu entnehmen ist, daß die Übertiefung sicherlich den Betrag von $74 \mathrm{~m}$ gegenüber dem heutigen Spiegel des Adriatischen Meeres erreicht hat ${ }^{1}$ ).

2) Dieser Feinkies zeichnet sich durch hohe Gleichförmigkeit aus $(U=2,2)$ und besteht aus kaum gerundeten Komponenten unter denen kristalline Gesteine weitaus vorwiegen. Meiner Meinung nach entstammt der Feinkies nahegelegenen Moränenablagerungen und gelangte durch subaquatische Rutschungen in das Seebecken (VENZo 1957).

1) Abb. 2 gibt die Lage der Felssohle nur angenähert wieder, da die im Bereich des Mußpunktes $\mathrm{E} 3$ ermittelte Tiefe nicht unbedingt die absolut größte ist. 


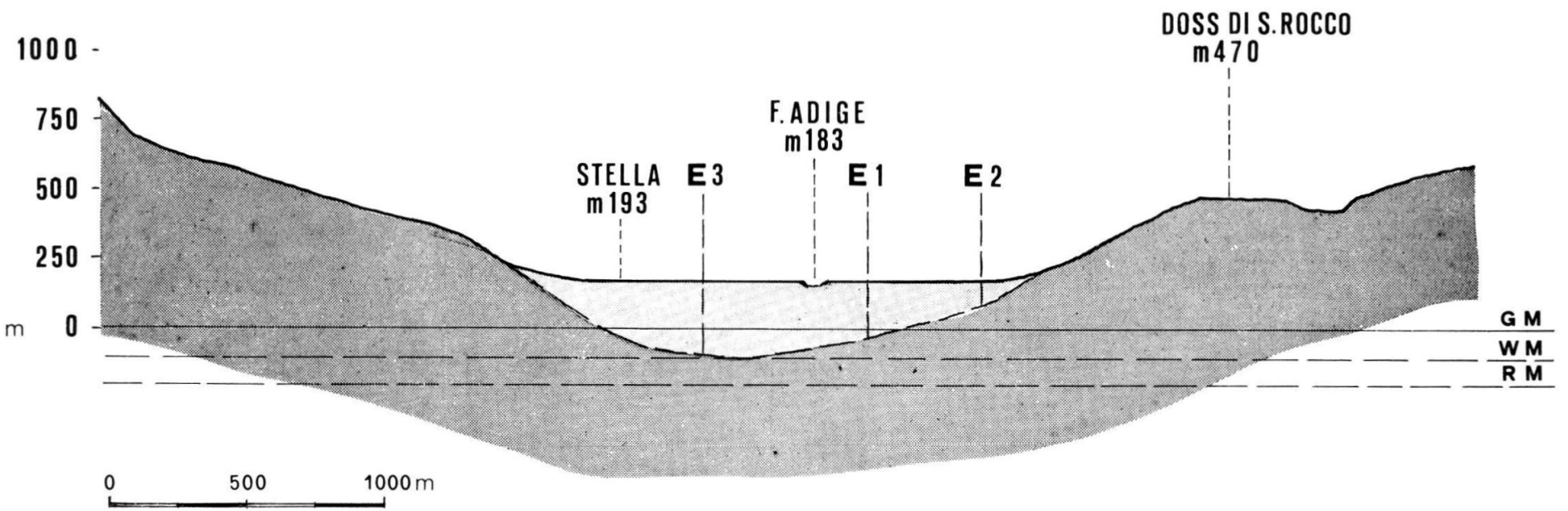

Abb. 3: Rekonstruktion des Verlaufes der Felssohle des Etschtales bei Trient nach den Ergebnissen der geoelektrischen Sondierungen $(1=$ Präquartäre Ablagerungen; 2 = Postglaziale Talverschüttung; $\mathrm{E}=$ Geoelektrische Meßpunkte; GM = Gegenwärtiger Meeresspiegel; WM = WürmKataglazialer Meeresspiegel; RM = Riß-Kataglazialer Meeresspiegel). 


\section{Die postglaziale geomorphologische Entwicklung}

An die in den vorhergehenden Abschnitten dargelegten Ergebnisse der Brunnenbohrungen und der geophysikalischen Untersuchungen lassen sich einige Deutungsversuche und Hypothesen betreffend die morphologische Entwicklung des Tales zur Zeit der postglazialen Verschüttung knüpfen ${ }^{3}$ ).

Außer der Tektonik ist meiner Meinung nach die morphologische Entwicklung im wesentlichen das Ergebnis zweier zusammenwirkender Faktoren, nämlich der intensiven Erosion der Wildbäche in den Seitentälern und des gleichzeitigen allmählichen Anstieges des Meeresspiegels.

Sicherlich mündeten beim Rückzug der Würmgletscher die von den Wildbächen Avisio und Fersina durchflossenen Seitentäler sowie der Graben von Ravina als Hängetäler hoch über der stark übertieften, eben erst eisfrei gewordenen Furche des Etschtales aus. Es ist anzunehmen, daß damals die Zuflüsse der Etsch eine intensive erosive Tätigkeit zu entfalten begannen, mit dem Bestreben, daß Gefälle zwischen den Mündungsstufen und ihrer Erosionsbasis, nämlich des Etschtales, auszugleichen. So gelangten an der Mündung der Seitentäler Wildbachkegel zur Ablagerung, die durch ihre Stauwirkung zur Bildung zwischengeschalteter Seebecken Anlaß gaben. Diese müssen langen Bestand gehabt haben, wie die bedeutende Mächtigkeit der schluffig-tonigen Sedimentfolge bezeugt, die die Bohrung P3 erschlossen hat.

Die starke glaziale Übertiefung des Etschtales steht meiner Meinung nach mit der Absenkung des Spiegels des Adriatischen Meeres in ursächlichem Zusammenhang, das seit jeher dessen Erosionsbasis darstellt. Die Spiegelsenkung betrug am Höhepunkt des WürmAnaglazials, vor 17.000 bis 18.000 Jahren (FAIRBRIDGE 1960), maximal etwa $100 \mathrm{~m}$ (nach einigen Autoren sogar $130 \mathrm{~m}$ ) gegenüber dem heutigen Stand ${ }^{4}$ ).

Daher ist anzunehmen, daß die nacheiszeitliche Talverschüttung, neben der Stauwirkung der Schwemmkegel der Seitenbäche, auch dem gleichzeitigen Spiegelanstieg des Adriatischen Meeres zuzuschreiben ist. Der Spiegelanstieg erfolgte allmählich und verhältnismäßig rasch, obschon nicht gleichmäßig, so daß die gegenwärtige Küstenlinie bereits vor etwa 6.000 Jahren erreicht war ${ }^{5}$ ).

Man darf demnach wohl behaupten, daß die quartären Meeresspiegelschwankungen, zumindest im Würm- und im Postglazial, unmittelbar die morphologische Entwicklung des Etschtales, auch im inneralpinen Anteil nördlich von Trient, beeinflußt haben.

5. Schriftenverzeichnis

FAIRBRidge, R. W. (1960): The Changing Level of the Sea. - Scientific American, 202, 5: 70-79; San Francisco.

VEnzo, G. A. (1957): Ricerche sulla serie lacustre e fluviale attraversata da pozzi trivellati nella zona di Trento. - Giornale di Geologia, 26: 1-16; Bologna.

3) Die Mächtigkeit der Talverschüttung betrüge im Raume von Trient $267 \mathrm{~m}$ unter der allerdings wenig wahrscheinlichen Annahme des Fehlens jeglicher Grundmoränenablagerung.

4) Eine noch bedeutendere Absenkung des Meeresspiegels als im Würm war im Riß-Glazial mit $-200 \mathrm{~m}$ gegenüber dem heutigen Stand zu verzeichnen.

5) Trotz nicht unbedeutender positiver wie negativer Schwankungen behielt das Meer seither im großen seine Spiegellage bei. 
